\title{
Original
}

\section{Factores asociados al desgaste profesional en los trabajadores del soporte vital básico del País Vasco: Un estudio cualitativo}

\author{
Factors associated with professional burnout among basic life support \\ caregivers in the Basque Country: A qualitative research
}

\author{
Sendoa Ballesteros Peña \\ SAMUR - Protección Civil. Bilbao. España \\ Recibido: 20-07-12 \\ Aceptado: 02-10-12

\section{Correspondencia} \\ Sendoa Ballesteros Peña \\ Bilbao SAMUR - Protección Civil \\ Parque de bomberos de Miribilla \\ Juan Carlos Gortázar 3 \\ 48003 Bilbao (España) \\ Tfno: 679.121.359 \\ Correo electrónico: sendoa.ballesteros@gmail.com
}

\section{Resumen}

Objetivos: Identificar factores de riesgo de desgaste profesional percibidos por los técnicos en emergencias sanitarias de las unidades de soporte vital básico del País Vasco.

Metodología: Investigación cualitativa realizada mediante análisis de discurso en ocho entrevistas individuales a trabajadores del soporte vital básico con puntuaciones compatibles con afectación por desgaste profesional en el Inventario de Burnout de Maslach. Las variables estructurales tenidas en cuenta para la elección de los participantes fueron la antigüedad profesional (superior o inferior a 10 años) y el centro de trabajo.

Resultados: Los trabajadores presentan disconformidad con la actual estructura organizacional del sistema de emergencias y falta de proyección y reconocimiento de su rol profesional. Además manifiestan un progresivo distanciamiento afectivo para con los pacientes, con quienes ha aumentado el número de enfrentamientos debidos a dificultades de entendimiento causadas por situaciones de inadecuación del uso de los recursos asistenciales.

Conclusiones: La estructura de organización del sistema de emergencias, el trato con el paciente y su familia y la situación profesional del técnico en emergencias son percibidos como elementos clave predisponentes de desgaste profesional.

Med Segur Trab (Internet) 2012; 58 (229) 294-302

Palabras clave: Agotamiento Profesional, Servicios Médicos de Urgencia, Salud Laboral. 


\section{Abstract}

Objective: To identify possible factors associated with professional burnout among basic life support caregivers in the Basque Country.

Method: A qualitative research was performed. We conducted an analysis of the discus of eight interpretive individual interviews with caregivers in basic life support units who presented scores compatible with burnout on the Maslach Burnout Inventory. The selection of participants was intentional.

Results: The data analysis found that ambulance personnel have disagreed with emergency system organizational structure and recognition of their professional role. They show a progressive emotional detachment to patients. An inadequate use of healthcare resources are increased the number of conflicts with patients and their families.

Conclusions: The organizational structure of emergency system, dealing with the patient and family and professional status of emergency medical technicians are key factors predisposing to burnout.

Med Segur Trab (Internet) 2012; 58 (229) 294-302

Key words: Professional Burnout, Emergency Medical Services, Occupational Health. 


\section{INTRODUCCIÓN}

Algunos autores han sugerido que los trabajadores de los servicios de emergencias experimentan más problemas de salud que la población activa general y que otros profesionales del ámbito sanitario ${ }^{1}$. Patologías como el síndrome de estrés post-traumático, problemas mentales, lesiones músculo-esqueléticas, enfermedades infecciosas e incluso accidentes mortales han sido relatados con frecuencia en la literatura biomédica sobre salud en el trabajo en el contexto de las emergencias sanitarias $^{2-4}$.

En las últimas décadas han adquirido especial relevancia las investigaciones dirigidas a la evaluación de alteraciones psicosociales en el ámbito laboral. El Síndrome de Desgaste Profesional (SDP), o burnout, es el resultado de un estado prolongado de estrés laboral, que afecta a personas cuya profesión implica una relación con los demás e interactúan cuidando y/o solucionando problemas ajenos 5 . Es por ello que los profesionales de la salud constituyen un colectivo especialmente vulnerable a este síndrome.

Un reciente estudio de corte epidemiológico llevado a cabo en el País Vasco ${ }^{6}$ revela que el 89\% de los Técnicos en Emergencias Sanitarias (TES) que desarrollan su trabajo en las Unidades de Soporte Vital Básico (USVB) presenta sintomatología compatible con el SDP. Sin embargo, a pesar de que los autores analizan la posible relación del síndrome con ciertas variables socio-laborales, tan sólo identifican una asociación de la patología con la antigüedad profesional, concluyendo sin lograr aislar otras causas etiológicas responsables de tan elevada prevalencia.

El objetivo del presente trabajo se centra en identificar factores de riesgo de desgaste profesional percibidos por los TES de las USVB del País Vasco.

\section{MATERIAL Y MÉTODOS}

Con la finalidad de acceder a una mayor profundidad de respuesta y a una mejor comprensión del fenómeno, se optó por utilizar una metodología cualitativa. La investigación se desarrolló a través de la técnica de entrevista individual semiestructurada. Esta estrategia supone un encuentro entre el investigador y los informantes, dirigido hacia la exploración de las perspectivas que estos últimos tienen respecto de sus vidas, experiencias o prácticas ${ }^{7}$.

En base a la literatura revisada, y a un pilotaje de entrevista previa realizado con tres sujetos, se elaboró un guión con preguntas abiertas que permitiese recoger información sobre características socioprofesionales y factores que pudiesen propiciar sentimientos de cansancio emocional, despersonalización o frustración profesional (dimensiones definitorias del $\mathrm{SDP}^{8}$.

Los participantes fueron seleccionados mediante muestreo intencional entre los TES trabajadores de las unidades de SVB integradas en la red de emergencias de Osakidetza que, tras invitarles a colaborar, accedieron voluntariamente. No se incluyó en el estudio al personal voluntario. Las variables estructurales que se tuvieron en cuenta en el diseño muestral fueron la antigüedad profesional en el sector (superior o inferior a 10 años) y el centro de trabajo (administración pública, sector privado $u$ organizaciones no gubernamentales/asociaciones sin ánimo de lucro). Con anterioridad al inicio de la fase de entrevistas, se proporcionó a los candidatos el Inventario de Burnout de Maslach -versión servicios sanitarios (MBI-HSS), con la finalidad de comprobar la presencia prevalente de indicadores de SDP 9 . Se consideraron puntuaciones compatibles SDP cuando los resultados en las subescalas "cansancio emocional" > 18; "despersonalización" $>5$ o "falta de realización profesional" $>40$.

Se realizaron entrevistas individuales hasta que ya no se obtuvo ninguna información nueva, lográndose la saturación con ocho TES. Los perfiles de los participantes se muestran en la tabla I. 
Tabla I: Perfiles de los trabajadores entrevistados

\begin{tabular}{cccccccc}
\hline E & Edad & Sexo & $\begin{array}{c}\text { Antigüedad } \\
\text { profesional }\end{array}$ & Sector trabajo & $\begin{array}{c}\text { Puntuación } \\
\text { CE }\end{array}$ & $\begin{array}{c}\text { Puntuación } \\
\text { DP }\end{array}$ & $\begin{array}{c}\text { Puntuación } \\
\text { FRP }\end{array}$ \\
\hline $\mathbf{1}$ & 50 & H & $\geq 10$ años & Público & 21 & 1 & 28 \\
$\mathbf{2}$ & 40 & H & $<10$ años & Público & 22 & 10 & 37 \\
$\mathbf{3}$ & 33 & H & $\geq 10$ años & ONG & 38 & 18 & 27 \\
4 & 36 & M & $\geq 10$ años & ONG & 33 & 12 & 29 \\
5 & 21 & H & $<10$ años & ONG & 16 & 6 & 36 \\
$\mathbf{6}$ & 28 & H & $\geq 10$ años & Privado & 19 & 17 & 35 \\
7 & 43 & H & $\geq 10$ años & Privado & 35 & 12 & 27 \\
$\mathbf{8}$ & 35 & M & $<10$ años & Privado & 34 & 10 & 40 \\
\hline
\end{tabular}

E: entrevistado; H: hombre; M: mujer; CE: Cansancio Emocional; DP: Despersonalización; FRP: Falta de Realización Profesional. NOTA: se consideran puntuaciones compatibles con Síndrome de Desgaste Profesional cuando CE $>18 ; D P>5$ ó FRP $<40$.

Al comienzo de cada sesión, se facilitó a los participantes un consentimiento informado donde se les explicaba la intencionalidad de la investigación, indicando que la entrevista, con el objetivo de evitar la pérdida de datos, sería grabada y transcrita y que toda la información derivada sería tratada confidencialmente, acorde a la Ley vigente de Protección de Datos de Carácter Personal ${ }^{10}$.

Finalizada la fase de entrevistas, se procedió a la lectura repetida de las trascripciones y notas de campo, elaborando fichas interpretativas para cada discurso. Mediante la comparación intertextual se identificaron significados comunes y temas interconectados. Como herramienta de apoyo para este análisis fue empleado el programa informático RQDA.

\section{RESULTADOS}

En el contexto estudiado, la problemática que condiciona las relaciones psicosociales y laborales generadoras de desgaste en el ámbito de las emergencias sanitarias extrahospitalarias puede estructurarse en torno a tres ejes fundamentales: la organización, el profesional y el usuario.

A continuación se detallan cada una de estas categorías, adjuntando citas literales ilustrativas que corroboran el análisis temático.

\section{El modelo organizacional del Sistema de Emergencias Médicas}

Un aspecto que aparece repetidamente en los diálogos es la percepción de que, si bien el trabajo que desempeñan los TES en ocasiones puede ser físicamente duro, se trata del cansancio psicológico el que con más fuerza afecta a su labor diaria. Sin embargo, la sobrecarga psicológica ha sido atribuida a dos factores bien diferenciados: el trato directo con el paciente y discrepancias con la estrategia organizacional del servicio.

"Más que la muerte en sí, me afecta el sufrimiento de la persona. No conseguir reanimar a un paciente que a nuestra llegada estaba en parada cardiaca no me impresiona tanto como ver a otra sufrir y poder hacer poco o nada por ella." (E7)

"No es el trabajo con los pacientes en si lo que sobrecarga psicológicamente, sino el enfoque que se da a la emergencia, la sensación de que realmente no se están haciendo emergencias y de estar muchas veces utilizando el servicio para otros menesteres." (E1)

A rasgos generales, los entrevistados han calificado como buena la relación con el resto de servicios de emergencia. En ocasiones se habla de diferencias de trato al 
interactuar con el personal de los recursos de distinto nivel (soporte vital avanzado o con enfermería), siendo calificada por algunos como clasista.

"Con gente del mismo sector y del mismo nivel no bay ningún problema, independientemente del color del que vaya vestido. Otras categorías profesionales marcan más su mando. Hay mucho clasismo. Especialmente entre médicos.” (E2)

"Las relaciones con el personal del hospital son muy variables. Depende mucho de con quién topes: a veces te escuchan cuando les cuentas lo que traes, y otras no te miran $y$ directamente te dicen el número de box al que debes acudir." (E6)

El abordaje de aspectos sobre las relaciones y comunicación existente con el Centro de Coordinación de Urgencias suscita un gran volumen de comentarios, mayoritariamente dirigidos en una misma línea. Es posible percibir un descontento generalizado con la gestión del Centro de Coordinación sobre los recursos de soporte vital básico.

Varios entrevistados aluden a la falta de información que transmiten cuando activan la unidad para acudir a una emergencia o las dificultades para ponerse en contacto con las operadoras.

"Es difícil hacer entender muchas cosas de la calle cuando hablas con alguien (del Centro Coordinador) que nunca ha trabajado en una ambulancia. Más que problemas de comunicación son problemas de empatía." (E4)

"Realmente no me quema hacer quince avisos en una jornada. Lo que me estresa es hacer quince avisos sin saber a lo que voy, porque el Centro Coordinador no me facilita información suficiente." (E2)

La escasez de personal en las ambulancias también constituyó una cuestión predominante, y se considera que una dotación compuesta de dos sanitarios puede resultar insuficiente.

"Dos personas es insuficiente. Muchas veces te faltan manos. Especialmente si el otro recurso más próximo está alejado y no puede acudir en tu ayuda.” (E5)

"Cargamos con pesos a menudo, y en situaciones de emergencia hacemos burradas. Con el tiempo el cuerpo se resiente. Un tercero en la ambulancia nos aborraría problemas de salud y aumentaría nuestra eficacia." (E7)

\section{El profesional sanitario de emergencias}

El sentimiento de vocación profesional ha representado una característica frecuente en los participantes. No obstante, algunos trabajadores hacen alusión al papel que juega el actual tipo de organización del servicio en el logro de sus expectativas profesionales. Las motivaciones personales que actualmente sostienen a los TES son diversas y poco homogéneas. El sueldo es el principal determinante, especialmente en el sector público, aunque también se presentan como factores el trabajo directo con las personas o la flexibilidad del horario laboral.

"Siento orgullo de ser Técnico, aunque la mayoría de gente no sabe lo que somos ni lo que hacemos realmente." (E8)

"Mi actividad profesional me encanta. Aunque considero que debería ser mejor el sistema de gestión del servicio [...]. Partiendo de la base de que en el sistema actual mi categoría de Técnico no se reconoce como tal, nopuedo decir que me sientaprofesionalmente realizado." (E6)

"No me encuentro profesionalmente satisfecho. Nos tienen infravalorados y poco aprovechados [...]. Después de una temporada en la que únicamente te motiva venir a trabajar por tu sueldo, te encuentras en una salida en la que bas sentido realmente que has ayudado a alguna persona que lo está pasando mal y te carga las pilas, recordándote por qué estás aquí." (E2) 
"Durante mucho tiempo bice voluntariado. Realmente creía en esto. Abora de este trabajo me fijo en el sueldo y la disponibilidad de tiempo libre que te permite." (E4)

Los cambios acaecidos en el marco formativo y profesional de los Técnicos en Emergencias Sanitarias suscitan esperanzas en el sector, deseando con ello que la figura del TES sea finalmente reconocida, puesto que resultan escasas las muestras de reconocimiento social y profesional que reciben.

"Me han llamado conductor, camillero, ayudante... Ya es hora de que nos llamen lo que somos: técnicos en emergencias." (E7)

"La regulación de la profesión a través de una formación oficial y reglada traerá grandes beneficios para la profesión, pero el problema se encuentra en la organización y gestión del servicio." (E3)

En la esfera personal, un considerable número de entrevistados ha manifestado que la tarea que desempeñan repercute, en menor o mayor grado, en sus relaciones personales, tanto de manera positiva como negativa. Dentro de las repercusiones positivas destacan el gran valor otorgado a la familia o los fuertes vínculos de amistad que se forman en el lugar de trabajo.

Pero también es preciso mencionar consecuencias negativas en sus relaciones, como la dificultad de conciliación entre la vida social y la laboral derivada de las condiciones propias del tipo de trabajo.

"Hay dias que al llegar a casa después de trabajar me siento afortunado. Trabajar con las desgracias ajenas te ayuda a valorar lo que tienes." (E7)

"Cuando comencé a trabajar en esto perdi el contacto con mi grupo de amigos. Trabajar a turnos, sin tener fines de semana libres hace difícil la vida social fuera de este mundo, por lo que abora, prácticamente sólo me relaciono con compañeros del gremio." (E8)

"Aunque siempre bay algún pequeño roce, una de las mejores cosas de este trabajo es que, el comer, dormir y pasar situaciones duras con los compañeros de trabajo hace que con muchos acabes teniendo una gran amistad." (E2)

\section{El usuario demandante del Sistema de Emergencias Médicas}

En cuanto a los factores relativos al contacto con el usuario del Sistema de Emergencias Médicas y su familia, los TES otorgan gran parte de la responsabilidad de su desgaste a las actitudes del demandante de asistencia. Si bien resultan habituales las actuaciones en las que los pacientes valoran la tarea de los sanitarios, los enfrentamientos o falta de entendimiento entre ambas partes, generada en muchas ocasiones por la percepción por parte del trabajador de un uso inadecuado de los recursos asistenciales, también representan situaciones que han ido en aumento.

"Con los pacientes y familiares, sobre todo en función de la zona geográfica, cada vez tenemos más enfrentamientos. A veces se abusa de nosotros y nos utilizan como taxi gratuito.” (E4)

"Tengo la sensación de ser un servicio de paquetería urgente." (E6)

"Habría que optimizar el aprovechamiento de los recursos de Soporte Vital Básico, que nos aprovecharan más como profesionales, y para eso sería necesario educar a la población." (E2)

Especialmente quienes ya llevan varios años ejerciendo en las emergencias extrahospitalarias han mostrado un distanciamiento afectivo o desensibilización hacia sus usuarios y a través del cual se permiten negar, desplazar o reprimir sus emociones, como estrategia de autoprotección. 
"El visualizar constantemente situaciones duras nos desensibiliza. No es nada bueno. Llegamos a hablar de comida incluso delante de pacientes que acaban de morir, y pensándolo fríamente es un poco... Pero eso lo hacemos todos." (E6)

"Puedo estar en el domicilio hablando con la familia tras la muerte de un familiar $y$ al bajar a la ambulancia desconectar completamente. E incluso a veces be sentido indiferencia." (E5)

"Yo no creo que sea menos sensible, pero sí menos vulnerable." (E1)

Por último, al abordar temas sobre la formación o entrenamiento de los profesionales para afrontar situaciones emocionalmente críticas (propias o ajenas) o técnicas de comunicación de malas noticias, todos los participantes coincidieron en la escasa o nula preparación que habían recibido, llegando a propiciar actitudes de evitación.

"No se nos forma en el control emocional, ni hacia las víctimas o familiares ni hacia nosotros mismos. La única técnica de debriefing que conocemos es colgar el uniforme $e$ irnos de cervezas.” (E3)

"No me siento capacitado para transmitir malas noticias o para consolar a los pacientes o sus familias. Si puedo evitar tener que hacerlo, lo evito.” (E7)

\section{DISCUSIÓN Y CONCLUSIONES}

El desgaste profesional es un proceso complejo y presenta aspectos de difícil valoración. El uso de metodología cualitativa permite investigar variables que no se tienen en cuenta en estudios cuantitativos y profundizar en las que ya se conocen.

Los métodos cuantitativos y cualitativos se deben considerar complementarios dado que, en ocasiones, dentro del continuo salud-enfermedad existen tanto elementos cuantificables como aspectos relacionados con la comprensión de los significados y los $\operatorname{contextos}^{11,12}$.

Algunas de las cuestiones más importantes de los factores de riesgo psicosocial percibidos por los TES se relacionan con características extrínsecas a su profesión. De tal modo, en este trabajo, los factores organizacionales del Sistema de Emergencias Médicas han representado los principales elementos implicados en la aparición de desgaste en el colectivo a estudio.

A pesar de que un considerable número de investigaciones han identificado la sobrecarga laboral como un importante estresor ${ }^{13,14}$, los participantes entrevistados no han percibido una sobrecarga de trabajo inusual en su actividad, pero sí apuntan hacia la necesidad de adecuar u optimizar los recursos de emergencia. Una queja prácticamente unánime hace referencia a la utilización de ambulancias de emergencia para valorar o resolver situaciones que a juicio de los trabajadores no revisten urgencia. La inadecuación del uso de los recursos sanitarios supone en nuestro país un problema común de difícil solución que parece extenderse también al ámbito extrahospitalario ${ }^{15}$.

Otros aspectos muy considerados por los entrevistados son el clima laboral y la relación entre compañeros y jefaturas, determinantes bien conocidos como propulsores de estrés crónico en el ámbito de la salud ${ }^{16,17}$.

La permanencia en el entorno de las emergencias sanitarias ocasiona que, derivado del importante desgaste emocional que sufren algunos profesionales, así como de las estrechas relaciones que surgen del contacto continuado con los compañeros de trabajo, lleguen a modificarse conductas que afectan a su vida personal ${ }^{18}$. El trabajo y la vida social no pueden ser concebidas como esferas separadas, sino relacionadas entre sí. La adaptación y la satisfacción dependen del equilibrio entre estas áreas, resultando la aparición de alteraciones del bienestar físico o mental en el caso de no conseguirlo ${ }^{19}$. 
Los TES muestran interés por la impresión que suscitan en otros colectivos sanitarios, sintiéndose en ocasiones poco valorados. Las preocupaciones relativas a la indefinición de la carrera profesional y las dudas acerca de los planes de estudios reglados han constituido un foco de atención entre los trabajadores de los recursos de SVB. La reciente regulación de la formación de los TES, enmarcada dentro de un ciclo formativo de grado medio $^{20}$, apunta a ser un punto de partida hacia la consecución del reconocimiento de esta figura dentro de las profesiones sanitarias.

En el tercer grupo de factores detectado depende directamente del usuario demandante de asistencia urgente, al constituir el principal objeto de atención profesional de los trabajadores de la salud.

Convivir en contacto con el sufrimiento y la muerte es un elemento importante generador de estrés identificado en la literatura ${ }^{21,22}$. Además, las actitudes del usuario y de su familia frente al profesional conforman una variable a considerar en la aparición y desarrollo de síntomas de desgaste. Con cierta frecuencia ha sido posible localizar conflictos entre el usuario o familia del demandante de asistencia y el equipo asistencial ${ }^{23}$, donde se produce una ruptura del entendimiento entre ambas partes, desencadenando situaciones de agotamiento emocional y de despersonalización como estrategia de afrontamiento del problema.

Finalmente, cabe mencionar que el hecho de que el personal sanitario de emergencias constituya un colectivo afectado por factores de riesgo psicosocial repercute no sólo sobre la calidad de vida del profesional y su entorno más próximo, sino que puede generar también importantes consecuencias en el rendimiento laboral, disminuyendo la calidad del servicio que presta ${ }^{24}$.

\section{REFERENCIAS BIBLIOGRÁFICAS}

1. Sterud T, Ekeberg $\varnothing$, Hem E. Health status in the ambulance services: a systematic review. BMC Health Serv Res. 2006; 6:82.

2. Alexander DA, Klein S. Ambulance personnel and critical incidents. Br J Psychiatry. 2001;178:76-81.

3. Van Der Ploeg E, Kleber RJ. Acute and chronic stressor among ambulance personnel: predictors of health symptoms. JOEM. 2003;60:40-46.

4. Bennett P, Williams Y, Page N, Hood K, Woollard M: Levels of mental health problems among UK emergency ambulance workers. Emerg Med J. 2004;21:235-236.

5. Gil Monte PR. Una nota sobre el concepto de burnout, sus dimendiones y estrategias de afrontamiento. Informació psicológica. 1991; 46:4-7.

6. Ballesteros Peña S, Lorrio Palomino S, Rollán Vallejos J. Desgaste profesional en los trabajadores del Soporte Vital Básico del País Vasco. Emergencias. 2012; 24(1): 13-18. Citado el 28/06/2012. Disponible en: http://www.semes.org/revista/vol24_1/5.pdf

7. Taylor SJ, Bodgan R. Introducción a los métodos cualitativos de investigación. Barcelona: Paidós, 1992.

8. González Rodríguez VM, González Correales R, de la Gándara Martín, JJ. El médico con burnout. Conceptos básicos y habilidades prácticas para el médico de familia. 1. ${ }^{a}$ ed. Madrid (España): IMC; 2004.

9. Seisdedos N. Manual MBI, Inventario Burnout de Maslach. Madrid: Ediciones TEA; 1997.

10. Ley Orgánica 15/1999, de 13 de diciembre, de Protección de Datos de Carácter Personal. Boletín Oficial del Estado. 298: 43088-43099. Citado el 5/05/2012. Disponible en http://www.boe.es/boe/dias/1999/12/14/ pdfs/A43088-43099.pdf

11. Fernández de Sanmamed MJ. Métodos y técnicas cualitativas en la investigación en atención primaria [editorial]. Aten Primaria 1999;23:453-4.

12. Faltermaier T. Why public health research needs qualitative approaches. Subjects and methods in change. Eur J Public Health 1997;7:357-63.

13. Flanagan NA, Flanagan TJ. An analysis of the relationship between job satisfaction and job stress in correctional nurses. Res Nurs Health 2002;25:282-94.

14. Miret C, Martínez Larrea A. El profesional en urgencias y emergencias: agresividad y burnout. Anales Sis San Navarra. 2010; 33 supl.1. 
15. Aranaz Andrés JM, Martínez Nogueras R, Gea Velázquez de Castro MT, Rodrigo Bartual V, Antón García P, Gómez Pajares F. ¿Por qué los pacientes utilizan los servicios de urgencias hospitalarios por iniciativa propia? Gac Sanit. 2006;20(4):311-5.

16. Lubrańska A. Organizational climate and burnout síndrome. Med Pr. 2011;62(6):623-31.

17. Della Valle E, De Pascale G, Cuccaro A, Di Mare M, Padovano L, Carbone U et al. Burnout: rising interest phenomenon in stressful workplace. Ann Ig. 2006;18(2):171-7.

18. Roth SG, Moore CD. Work-family fit: the impact of emergency medical services work on the family system. Prehosp Emerg Care. 2009;13(4):462-8.

19. Regehr C, Dimitropoulos G, Bright E, George S, Henderson J. Behind the brotherhood: rewards and challenges for wives of firefighters. Family Relations. 2005;54:423-35.

20. Real Decreto $1397 / 2007$, de 29 de octubre, por el que se establece el título de Técnico en Emergencias Sanitarias y se fijan sus enseñanzas mínimas. Boletín Oficial del Estado 282, del 24 de noviembre de 2007. Citado el 13/05/2012. Disponible en: http://www.boe.es/boe/dias/2007/11/24/pdfs/A4817848211.pdf

21. Zambrano Plata GE. Estresores en las unidades de cuidado intensivo. Aquichan. 2006;6(1):156-169.

22. Simón García MJ, Blesa Malpica AL, Bermejo Pablos C, Calvo Gutierro MA, Gómez De Enterría Pérez C. Estresores laborales y satisfacción en la enfermería de una unidad de críticos. Enferm Intensiva. 2005; 16:3-14

23. Alonso Pérez de Ágreda JP, Febrel Bordejé M, Huelin Domeco de Jarauta J. Factores asociados a la derivación inadecuada entre atención primaria y especializada: estudio cualitativo en médicos de atención primaria. Gac Sanit 2000;14(2):122-130.

24. Deckard G, Meterko M, Field D. Physician burnout: an examination of personal, professional, and organizational relationships. Med Care. 1994;32(7):745-5.

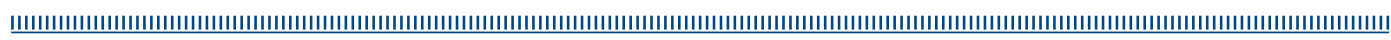

